\title{
ASEP of MIMO System with MMSE-OSIC Detection over Weibull-Gamma Fading Channel Subject to AWGGN
}

\author{
Keerti Tiwari, ${ }^{1}$ Davinder S. Saini, ${ }^{2}$ and Sunil V. Bhooshan ${ }^{1}$ \\ ${ }^{1}$ Department of Electronics and Communication Engineering, Jaypee University of Information Technology, \\ Waknaghat, Solan 173234, India \\ ${ }^{2}$ Department of Electronics and Communication Engineering, Chandigarh College of Engineering and Technology, \\ Chandigarh 160019, India \\ Correspondence should be addressed to Keerti Tiwari; krt.tiwari@gmail.com
}

Received 14 November 2015; Revised 28 January 2016; Accepted 15 February 2016

Academic Editor: Pantelis-Daniel Arapoglou

Copyright ( $\odot 2016$ Keerti Tiwari et al. This is an open access article distributed under the Creative Commons Attribution License, which permits unrestricted use, distribution, and reproduction in any medium, provided the original work is properly cited.

Ordered successive interference cancellation (OSIC) is adopted with minimum mean square error (MMSE) detection to enhance the multiple-input multiple-output (MIMO) system performance. The optimum detection technique improves the error rate performance but increases system complexity. Therefore, MMSE-OSIC detection is used which reduces error rate compared to traditional MMSE with low complexity. The system performance is analyzed in composite fading environment that includes multipath and shadowing effects known as Weibull-Gamma (WG) fading. Along with the composite fading, a generalized noise that is additive white generalized Gaussian noise (AWGGN) is considered to show the impact of wireless scenario. This noise model includes various forms of noise as special cases such as impulsive, Gamma, Laplacian, Gaussian, and uniform. Consequently, generalized Q -function is used to model noise. The average symbol error probability (ASEP) of MIMO system is computed for 16-quadrature amplitude modulation (16-QAM) using MMSE-OSIC detection in WG fading perturbed by AWGGN. Analytical expressions are given in terms of Fox-H function (FHF). These expressions demonstrate the best fit to simulation results.

\section{Introduction}

In multiple-input multiple-output (MIMO) systems, the role of spatial multiplexing (SM) and spatial diversity is to provide high data rate and reliable communication, respectively. However, a tradeoff occurs between diversity and multiplexing for multiple access channels [1]. The demand to achieve high data rate is increasing for next generation communication systems. Using diversity, a simple detection can be formed at the cost of capacity reduction. Thus, spatial multiplexing is recommended for achieving significant capacity gain with improved transmission rate. However, demultiplexing at the receiver is still an issue for efficient system design.

A variety of detection techniques exist in literature to improve the system performance [2-7]. Zero forcing (ZF) and minimum mean square error (MMSE) are simple detection techniques although they lead to the reduced error performance $[3,4]$. Maximum likelihood (ML) detection provides an optimal error rate performance. However, the hardware complexity increases with the increase in transmitter antennas and modulation order. Therefore, ordered successive interference cancellation (OSIC) is recommended with MMSE for improving the error rate performance [5]. In [5-8], efficient approaches have been investigated to develop OSIC and to reduce the complexity of receiver for VerticalBell Laboratories Layered Space-Time (V-BLAST) system. In [9], antenna selection has been used for OSIC detection to enhance the error rate performance. Also, a near-optimal selection technique has been proposed to decrease the complexity without substantial performance reduction. Different detection ordering such as signal-to-noise ratio (SNR) based, column norm based and, signal-to-interference plus noise ratio (SINR) based ordering has been investigated. It is examined that postdetection SINR based ordering achieves the best performance among all three ordering techniques [10]. Hence, MMSC-OSIC detection is used in this paper to achieve the improved error rate performance with adaptable 
complexity level in composite fading scenario. Identifying an efficient detection technique is still under investigation in MIMO systems.

Various small scale multipath channel models including Rayleigh, Rician, Nakagami-m, and Weibull have been recommended to analyze the wireless system performance. Large scale fading can be modeled using log-normal (LN) or Gamma distribution. Both the small scale and large scale fading effects can be observed simultaneously due to rapidly changing wireless environment. Therefore, channel modeling of composite fading which comprises multipath as well as shadowing effects is imperative to figure out. Also, it is important to resolve a number of practical problems with interference effects in MIMO wireless communications. The composite channel models such as RayleighLN, shadowed-Rician, Gamma-Gamma, K, generalized-K, correlated shadowed- $\kappa-\mu$ [11-17], and comparatively newfangled Weibull-Gamma (WG) [18, 19] are widely used to incorporate both small scale fading and shadowing. Gamma models are simpler and more accurate than LN models and hence preferred in recent approaches. The WG composite distribution is appropriate for MIMO system design in the present wireless scenario due to its extensive flexibility, experimental efficiency, and analytical conformity. Weibull fading model is adaptive for modeling severe and nonsevere multipath fading conditions, Gamma model is used for shadowing, and collectively WG composite fading model is formed to quantify both the effects. WG fading model is a generic model and it includes Rayleigh-Gamma or $\mathrm{K}$ and exponential-Gamma distributions as its special cases. Consequently, this model approximates several other fading models [11, 18].

Both higher and lower modulation orders have been considered in multipath fading with the existence of AWGN [20-22]. However, the deviation of actual noise is possible; therefore, generic noise model is required. In power line communication (PLC), the system performance is extensively affected by additive and multiplicative power line noises. Further, the additive noise is categorized into background noise and impulsive noise. The background noise and impulsive noise models follow Nakagami-m and Middleton class A distribution, respectively, although the multiplicative PLC noise induces fading in the received signal power. Also, the system error rate performance has been evaluated in the presence of such noise scenarios [23-25]. The generalized Gaussian distribution (GGD) is the emerging research interest for modeling different noise effects. This generic noise model considers various forms of noise such as impulsive, Gamma, Gaussian, Laplacian [26]. Rectangular quadrature amplitude modulation (QAM) is defined by a combination of in-phase and quadrature phase pulse amplitude modulation (PAM) signals. This modulation technique has been used to compute average symbol error probability (ASEP) using Gaussian Qfunction in composite fading scenario perturbed by additive white generalized Gaussian noise (AWGGN) [27].

To the best of our knowledge, the MIMO system performance has not been evaluated for composite WG fading channel with the consideration of generalized noise. To improve the MIMO system performance spatial multiplexing
(SM) is used with efficient detection technique, that is, MMSE-OSIC. The expressions derived for ASEP in [27] are analyzed again which were previously limited to single-input single-output (SISO) system. To achieve the high data rate of the wireless link, higher order modulation techniques are preferred, although they are less flexible to noise and interference. Exact analytical expressions are computed in terms of Fox-H function (FHF) for 16-QAM in SM-MIMO WG fading subject to AWGGN. Two special cases of AWGGN, namely, AWGN and Laplacian noise, are considered. AWGN is popularly known and Laplacian noise has also achieved attention in the signal processing and wireless systems to model impulsive noise. In addition, the variations of fading and shadowing parameters are also illustrated.

The rest of the paper is organized as follows. In Section 2, system model and proposed detection technique are described. Section 3 provides the simulation results and analysis for ASEP of SM-MIMO in WG fading subject to generic noise along with specified detection technique. Finally, the paper is concluded in Section 4.

\section{System and Channel Model}

Consider a MIMO system having $N_{r} \times N_{t}$ antennas, where $N_{t}$ and $N_{r}$ denote the number of transmit and receive antennas, respectively. $X$ is the transmit signal modulated by $16-\mathrm{QAM}$ and multiplied by a composite flat fading channel envelope $\mathscr{H}$. Previously, the noise is generally considered as AWGN. However, the AWGGN noise $\mathcal{N}$ is assumed here with zero mean and variance $\sigma^{2}$. MIMO system model is represented as

$$
Y=\mathscr{H} X+\mathcal{N}
$$

The probability density function (PDF) of the AWGGN noise is described in [28, Equation (6.2)] over $n \in \mathfrak{R}$ as

$$
\mathscr{P}_{\mathcal{N}}\left(n \mid m_{n}, \sigma, \eta\right)=\frac{\eta \psi}{2 \Gamma(1 / \eta)} \exp \left(-\psi^{\eta}\left|n-m_{n}\right|^{\eta}\right),
$$

where $\eta$ and $m_{n}$ denote shaping parameter of $\mathcal{N}$ and mean, respectively $\left(\eta \in \mathfrak{R}^{+}, \boldsymbol{m}_{n} \in \mathfrak{R}^{+}\right)$. Furthermore, the coefficient $\psi$ can be defined by normalizing the noise power using normalizing coefficient $\psi_{0}$ with respect to $\eta$ as

$$
\psi=\frac{\psi_{0}}{\sigma}=\sqrt{\frac{2 \Gamma(3 / \eta)}{N_{0} \Gamma(1 / \eta)}},
$$

where $\Gamma(\cdot)$ denotes Gamma function, $\psi_{0}=\sqrt{\Gamma(3 / \eta) / \Gamma(1 / \eta)}$, $\sigma^{2}=E\left[\mathcal{N}^{2}\right]-m_{n}{ }^{2}=N_{0} / 2, N_{0}$ is the power per positive frequency of AWGGN, and $E[\cdot]$ is expectation operator.

The random variable of AWGGN distribution rigorously depends on its shaping parameter $\eta$. This distribution reveals a superior fit to the quantified noise statistics with the changing physical channel conditions and forms various noise categories as special cases of AWGGN. For $\eta=2, \eta=1$, $\eta=0.5$, and $\eta=0$, it characterizes the prominent Gaussian, Laplacian, Gamma, and impulsive noise, respectively. Consequently, for $\eta=1 / 2$ and $\eta=1 / 3$, the statistical properties 
and accurate simulation technique have been developed in the presence of AWGGN [29].

The PDF of the received signal envelope $\chi$ given in [30] is simplified to form WG distribution defined over $x \in(0, \infty)$ and represented as

$$
\begin{aligned}
& \mathscr{P}_{\chi}(x)=\frac{2}{\Gamma m}\left[\frac{(m+1) \Gamma(1+1 / k)}{\Omega}\right]^{m} \\
& \cdot x^{2 m-1} \Gamma\left[\left(1-\frac{m}{k}\right), 0,\left(\frac{(m+1) \Gamma(1+1 / k)}{\Omega}\right)^{m} x^{2}, \frac{1}{k}\right],
\end{aligned}
$$

where $\Gamma(\cdot, \cdot, \cdot, \cdot)$ is extended incomplete Gamma function, which is given as $\Gamma(\xi, s, a, \mu)=\int_{s}^{\infty} x^{\xi-1} \exp \left(-x-a x^{-\mu}\right) d x$, $\left(s \in \mathfrak{R}^{+}\right),(\xi, a, \mu) \in \mathbb{C}[28$, Equation (6.2)]. $k$ and $m$ denote fading figure and shadowing shaping parameter, respectively, where $0.5 \leq k<\infty, 0 \leq m<\infty$, and $\Omega=E\left[\chi^{2}\right]$ $(0 \leq \Omega<\infty)$ is the average power of the received signal envelope.

The SNR $\gamma$ for received symbols in the presence of AWGGN follows WG PDF, which is defined over $\gamma \in(0, \infty)$. The average SNR per symbol is $\bar{\gamma}=E[\gamma]=E\left[\chi^{2}\right] E_{x} / N_{0}$, where $E_{x}$ is the energy of transmitted symbols. By exchanging variables, PDF is represented in the form of SNR as

$$
\begin{aligned}
& \mathscr{P}_{\gamma}(\gamma)=\frac{1}{\Gamma m}\left[\frac{(m+1) \Gamma(1+1 / k)}{\bar{\gamma}}\right]^{m} \\
& \cdot \gamma^{m-1} \Gamma\left[\left(1-\frac{m}{k}\right), 0,\left(\frac{(m+1) \Gamma(1+1 / k)}{\bar{\gamma}}\right)^{m} \gamma, \frac{1}{k}\right] .
\end{aligned}
$$

Equation (5) can be expressed in terms of the FHF using [28, Equation (6.22)], [31, Equations (2.1.4), (2.1.5) and (2.1.11)] and simplifying [32, Equation (8)] as

$$
\begin{aligned}
& \mathscr{P}_{\gamma}(\gamma) \\
& \quad=\frac{1}{\Gamma m \gamma} H_{0,2}^{2,0}\left[\left.\frac{(m+1) \Gamma(1+1 / k) \gamma}{\bar{\gamma}}\right|_{(1,1 / k),(m, 1)} ^{-}\right],
\end{aligned}
$$

where

$$
\begin{aligned}
& H_{p, q}^{m, n}\left[\left.z\right|_{\left(b_{k}, B_{k}\right)_{1, q}} ^{\left(a_{j}, A_{j}\right)_{1, p}}\right]=\frac{1}{2 \pi i} \\
& \quad \cdot \int_{c}^{-} \frac{\prod_{k=1}^{m} \Gamma\left(b_{k}, B_{k} s\right) \prod_{j=1}^{n} \Gamma\left(1-a_{j}, A_{j} s\right)}{\prod_{k=n+1}^{p} \Gamma\left(a_{j}, A_{j} s\right) \prod_{k=m+1}^{q} \Gamma\left(1-b_{k}, B_{k} s\right)} z^{-s} d s,
\end{aligned}
$$

where $H_{p, q}^{m, n}(\cdot)$ is FHF defined in [31, Equation (1.1.1)], [33] and $c$ represents Mellin-Barnes contour.

\section{MMSE-OSIC Detection}

MMSE detection technique maximizes the postdetection SINR by minimizing mean-square error (MSE). In OSIC, SINR based ordering improves the performance of linear detection technique by maximizing SINR. This technique maintains the low complexity for designing hardware. It holds number of linear receivers in which each receiver classifies one of the parallel data streams with detected signal components. These signal components are successively canceled from the received signal at each stage [34].

The MMSE detection technique explained in [10] offers the 1st estimated stream with the 1st row vector of the MMSE weight matrix $W_{\text {MMSE }}$ which is given by

$$
W_{\text {MMSE }}=\left(\mathscr{H}^{\dagger} \mathscr{H}+N_{0} I\right)^{-1} \mathscr{H}^{\dagger},
$$

where $(\cdot)^{\dagger}$ denotes the Hermitian operator. In MMSE detection, to determine the required statistical information of $N_{0}$, the $i$ th row vector $w_{i, \mathrm{MMSE}}$ of $W_{\mathrm{MMSE}}$ is obtained by

$$
w_{i, \mathrm{MMSE}}=\underset{w=\left(w_{1}, w_{2}, \ldots, w_{N_{t}}\right)}{\arg \max } \frac{\left|w h_{i}\right|^{2} E_{x}}{\sum_{j=1, j \neq i}^{N_{t}} E_{x}\left|w h_{i}\right|^{2}+N_{0}\|w\|^{2}},
$$

where $h_{i}$ is the $i$ th column vector of the channel matrix and $\|\cdot\|$ is the Frobenius norm of matrix. $x_{(i)}$ denotes the $i$ th order detected symbol which depends on the order of detection; hence, this symbol may be different from the transmit signal at the $i$ th antenna. The sliced value of $x_{(i)}$ is given by $\widehat{x}_{(i)}$. The remaining signal is represented by

$$
\widetilde{Y}=Y-\sum_{j=1}^{i-1} \widehat{x}_{j} h_{j}
$$

Assume that $\hat{x}_{j}, j=1,2, \ldots, i-1$, is accurately generated. When $x_{(1)}=\widehat{x}_{(1)}$ it means the interference is canceled and $x_{(2)}$ can be estimated. When $x_{(1)} \neq \widehat{x}_{(1)}$ then error propagation takes place. The entire performance of OSIC technique is affected by the order of detection. The erroneous outcomes in the previous stages cause the error propagation.

Primarily, signals containing a higher postdetection SINR are detected in SINR based ordering. The linear MMSE detection with the postdetection SINR is represented by

$$
\begin{array}{r}
\operatorname{SINR}_{i}=\frac{E_{x}\left|w_{i, \mathrm{MMSE}} h_{i}\right|^{2}}{\sum_{r \neq i} E_{x}\left|w_{i, \mathrm{MMSE}} h_{r}\right|+N_{0}\left\|w_{i, \mathrm{MMSE}}\right\|^{2}}, \\
i=1,2, \ldots, N_{t} .
\end{array}
$$

Once the selection of the $N_{t}$ SINR values using $W_{\text {MMSE }}$ is completed, the corresponding layer with the highest SINR is chosen. The second detected symbol is selected by canceling the interference due to first detected symbol from the received signals. If $r$ th symbol is canceled first, then $\mathscr{H}$ of (8) is transformed into (12) by deleting the channel gain vector as per $r$ th symbol:

$$
\mathscr{H}^{(r)}=\left[\begin{array}{lllllll}
h_{1} & h_{2} & \cdots & h_{r-1} & h_{r+1} & \cdots & h_{N_{t}}
\end{array}\right] .
$$

Again, $W_{\text {MMSE }}$ is calculated after substituting $\mathscr{H}$ of (8) by (12). Then, $N_{t}-1$ SINR values (i.e., $\left.\left(\operatorname{SINR}_{i}\right)_{i=1, i \neq r}^{N_{t}}\right)$ are computed by selecting the symbol containing highest SINR. After canceling the next symbol with the highest SINR, the same process is continued with the remaining signal. The total number of calculated SINR values is generated by $\sum_{j=1}^{N_{t}} j=$ $N_{t}\left(N_{t}+1\right) / 2$. 
The OSIC technique can offer diversity order greater than $N_{r}-N_{t}+1$ for all symbols. Following the ordering approach, the diversity order of the first detected symbol is also greater than $N_{r}-N_{t}+1$. Nevertheless, the diversity order of remaining symbols depends on whether the previously detected symbols are exact or not. Suppose all the symbols are exact; then, the diversity order of the $i$ th detected symbol is $N_{r}-N_{t}+i$. The $i$ th detected symbol is different from the one transmitted from the $i$ th transmit antenna. Since the ordering is based on SINR for MMSE detection, therefore, (11) is used to improve the ASEP performance.

\section{Average Symbol Error Probability for $M-Q A M$}

The symbol error probability (SEP) has been given in [35, Equation (10)] for QAM in the presence of AWGN. The formation of $M$-QAM signal constellation is given by two independent in-phase and quadrature $M$-ary PAM signals, where $M_{I}$-ary PAM and $M_{Q^{2}}$-ary PAM are in-phase and quadrature signals, respectively, and $M=M_{I} M_{\mathscr{Q}}$. Given that, GGD and Gaussian distribution demonstrate the identical symmetry properties. According to [27], the identical symmetry properties can be used to define SEP of $M$-QAM, given as

$$
\begin{aligned}
\mathscr{P}(\mathrm{SEP}) \\
=2\left(1-\frac{1}{M_{I}}\right) \mathscr{Q}_{\eta}\left(\mathscr{A}_{I}\right)+2\left(1-\frac{1}{M_{\mathscr{Q}}}\right) \mathscr{Q}_{\eta}\left(\mathscr{A}_{\mathscr{Q}}\right) \\
\quad-4\left(1-\frac{1}{M_{I}}\right)\left(1-\frac{1}{M_{\mathscr{Q}}}\right) \mathscr{Q}_{\eta}\left(\mathscr{A}_{I}\right) \mathscr{Q}_{\eta}\left(\mathscr{A}_{\mathscr{Q}}\right),
\end{aligned}
$$

where $\mathscr{A}_{I}=\mathscr{D}_{I} / \sigma, \mathscr{A}_{Q}=\mathscr{D}_{Q} / \sigma$, and $\mathscr{D}_{I}$ and $\mathscr{D}_{Q}$ denote the decision distances for in-phase and quadrature phase components, respectively. $Q_{\eta}(\cdot)$ is generalized-Q function for $x \geq 0$ defined in [32] as

$$
Q_{\eta}(x)=\frac{\eta \psi_{0}}{2 \Gamma(1 / \eta)} \int_{x}^{\infty} e^{-\psi_{0}^{\eta} t^{\eta}} d t
$$

In $[32, A .5]$, the representation of (14) in the form of FHF using [33, Equation $(8.3 .2 / 21),($ A.4) $]$ is given as

$$
Q_{\eta}(x)=\frac{1}{2 \Gamma(1 / \eta)} H_{1,2}^{2,0}\left[\left.\psi_{0}^{\eta}|x|^{\eta}\right|_{(1 / \eta, 1),(0,1)} ^{(1,1)}\right] .
$$

The ASEP is obtained by averaging the conditional SEPs in (13) under slow fading conditions over the PDF of $\gamma$. Then, $\mathscr{P}_{\gamma}(\gamma)$ is represented by

$$
\begin{aligned}
\mathscr{P r}\left(S_{e}\right)= & 2\left(1-\frac{1}{M_{I}}\right) \mathfrak{T}\left(\mathscr{A}_{I}\right) \\
& +2\left(1-\frac{1}{M_{\mathscr{Q}}}\right) \mathfrak{I}\left(\mathscr{A}_{\mathscr{Q}}\right) \\
& -4\left(1-\frac{1}{M_{I}}\right)\left(1-\frac{1}{M_{\mathscr{Q}}}\right) \mathfrak{J}
\end{aligned}
$$

where

$$
\begin{aligned}
\mathfrak{T}(x) & =\int_{0}^{\infty} \mathscr{Q}_{\eta}(\sqrt{\gamma} x) \mathscr{P}_{\gamma}(\gamma) d \gamma, \\
\mathfrak{J} & =\int_{0}^{\infty} \mathscr{Q}_{\eta}\left(\sqrt{\gamma} \mathscr{A}_{I}\right) \mathscr{Q}_{\eta}\left(\sqrt{\gamma} \mathscr{A}_{\mathscr{Q}}\right) \mathscr{P}_{\gamma}(\gamma) d \gamma .
\end{aligned}
$$

It is difficult to formulate $\mathfrak{\mathfrak { I }}(\cdot)$ and $\mathfrak{\Im}$ using the conventional expressions of WG distribution and GGD. Therefore, alternative expressions (6) and (15) are used to compute simplified analytical expressions for $\mathfrak{T}(\cdot)$ and $\mathfrak{S}$ and then resulting expression for the ASEP. In (17), $\mathfrak{I}(x)$ consists of an integral including the product of two FHFs which is comparable to that of [32] considering the normalized value of fading shaping factor and severity of shadowing. Unlike [32], we prefer an efficient SINR based ordering for MMSE detection to improve the error rate performance of MIMO system. Using [27] and [31, Equation (1.1.1)], $\mathfrak{I}(x)$ can be represented in the form of FHF by a closed form expression given as

$$
\begin{aligned}
& \mathfrak{T}(x)=\frac{1}{\eta \Gamma(1 / \eta) \Gamma m} \\
& \cdot H_{2,3}^{2,2}\left[\left.\frac{(m+1) \Gamma(1+1 / k)}{x^{2} \psi_{0}^{2} \bar{\gamma}}\right|_{(1,1 / k),(m, 1),(0,2 / \eta)} ^{(1-1 / \eta, 2 / \eta),(1,2 / \eta)}\right], \\
& \mathfrak{J}=\frac{1}{2 \eta \Gamma\left(1 / \eta^{2}\right) \Gamma m} H_{2,1 ; 0,2 ; 1,2}^{0,220 ; 2,0}\left[\frac{(m+1) \Gamma(1+1 / k)}{\mathscr{A}_{I}^{2} \psi_{0}^{2} \bar{\gamma}},\right. \\
& \left.\left.\quad\left(\frac{\mathscr{A}_{Q}}{\mathscr{A}_{I}}\right)^{\eta}\right|_{(1,1 / k),(m, 1),(1,1),(1 / \eta, 1),(0,1)} ^{(1-1 / \eta ; 2 / \eta, 1),(1 ; 2 / \eta, 1),(0 ; 2 / \eta, 1)}\right] .
\end{aligned}
$$

Substituting (6) and (15) in (18), an integral which includes the product of three FHFs is used to describe (20). Then, using [36, Equation (2.3)], $\mathfrak{J}$ is expressed in terms of the FHF of two variables known as the bivariate Fox-H function (BFHF).

Substituting (19) and (20) in (16), the ASEP of $M$-QAM is computed. This ASEP expression is given for rectangular $\left(M_{I} \neq M_{\mathscr{Q}}\right)$, square $\left(M_{I}=M_{\mathscr{Q}}\right)$ QAM in arbitrary WG fading with AWGGN. Consequently, it maintains substantial range of noise and fading parameters. The commonly considered noise cases of AWGGN in composite fading scenario are as follows.

Case 1 (WG fading with Laplacian noise). The first special case of AWGGN emerges when $\eta=1$, and the noise is considered Laplacian. Taking $\eta=1, \mathfrak{I}(x)$ is represented as

$\mathfrak{I}(x)$

$$
=\frac{1}{\Gamma m} H_{2,3}^{2,2}\left[\left.\frac{(m+1) \Gamma(1+1 / k)}{2 x^{2} \bar{\gamma}}\right|_{(1,1 / k),(m, 1),(0,2)} ^{(0,2),(1,2)}\right] .
$$

Using [31, 36], FHF and BFHF functions are well explored and utilized to make a simplified form of (21) by reducing number of terms in $H_{., \cdot}^{\cdot}(\cdot)$ as

$$
\mathfrak{T}(x)=\frac{1}{\Gamma m} H_{1,2}^{2,1}\left[\left.\frac{(m+1) \Gamma(1+1 / k)}{2 x^{2} \bar{\gamma}}\right|_{(1,1 / k),(m, 1)} ^{(1,2)}\right] .
$$


Similarly, $\mathfrak{J}$ can be written as

$$
\begin{aligned}
\mathfrak{S} & =\frac{1}{2 \Gamma m} H_{2,1 ; 0,2 ; 1,2}^{0,2 ; 2,0 ; 0}\left[\frac{(m+1) \Gamma(1+1 / k)}{2 \mathscr{A}_{I}^{2} \bar{\gamma}},\right. \\
& \left.\left.\left(\frac{\mathscr{A}_{Q}}{\mathscr{A}_{I}}\right)\right|_{(1,1 / k),(m, 1),(1,1),(1,1),(0,1)} ^{(0 ; 2,1),(1 ; 2,1),(0 ; 2,1)}\right] .
\end{aligned}
$$

Using [36, Equation (1.1)], for the description of $\mathrm{BHFH}$ and [28, Equations (6.29) and (6.42)], $\mathfrak{\Im}$ is represented as

$$
\mathfrak{S}=\frac{1}{2 \Gamma m} H_{1,2}^{2,1}\left[\left.\frac{(m+1) \Gamma(1+1 / k)}{2\left(\mathscr{A}_{I}^{2}+\mathscr{A}_{Q}^{2}\right) \bar{\gamma}}\right|_{(1,1 / k),(m, 1)} ^{(1,2)}\right] .
$$

Equations (22) and (24) are used to calculate ASEP when Laplacian noise is present.

Case 2 (WG fading with AWGN). For $\eta=2$, (19) and (20) can be rearranged to find the ASEP in AWGN environment. Again, the expressions for $\mathfrak{T}(x)$ and $\mathfrak{J}$ are reduced as

$$
\begin{aligned}
\mathfrak{I}(x) & =\frac{1}{2 \sqrt{\pi} \Gamma m} \\
\cdot & H_{2,3}^{2,2}\left[\left.\frac{(m+1) \Gamma(1+1 / k)}{2 x^{2} \bar{\gamma}}\right|_{(1,1 / k),(m, 1),(0,1)} ^{(1 / 2,1)(1,1)}\right], \\
\mathfrak{J} & =\frac{1}{14.5 \Gamma m} H_{2,1 ; 0,2 ; 1,2}^{0,2 ; 2,02,0}\left[\frac{(m+1) \Gamma(1+1 / k)}{2 \mathscr{A}_{I}^{2} \bar{\gamma}},\right. \\
& \left.\left.\left(\frac{\mathscr{A}_{Q}}{\mathscr{A}_{I}}\right)^{2}\right|_{(1,1 / k),(m, 1),(1,1),(1 / 2,1),(0,1)} ^{(1 / 2 ; 1,1),(1 ; 1,1),(0 ; 1,1)}\right] .
\end{aligned}
$$

Here, $m \rightarrow \infty$ eliminates the effect of shadowing. When $m \rightarrow \infty$, WG distribution follows Weibull distribution and $m \rightarrow \infty, k=1$ converted WG distribution into Rayleigh distribution [30, Table 1]. Thus, the fading scenario can be changed by setting the parameters $m$ and $k$.

\section{Simulation Results and Analysis}

To evaluate the MIMO system performance, 16-QAM modulation is used as a function of SNR for the generalized case of noise. Therefore, distinct values of $\eta$ and arbitrary values of $m$ and $k$ are taken into consideration. The in-phaseto-quadrature phase decision distance ratio is represented as $R_{\mathrm{ddr}}=\mathscr{D}_{I} / \mathscr{D}_{Q}=\mathscr{A}_{Q} / \mathscr{A}_{I}$. For this case, the average total energy per symbol $E_{T}$ is given as $E_{T}=10.5 \bar{\gamma} \mathscr{D}_{I}^{2}+$ $2.5 \bar{\gamma} \mathscr{D}_{Q}{ }^{2}=0.5\left(21+5 R_{\mathrm{ddr}}{ }^{2}\right) \bar{\gamma}_{\mathscr{D}_{I}}^{2}$ and hence $E_{T} / \sigma^{2}=$ $0.5\left(21+5 R_{\mathrm{ddr}}{ }^{2}\right) \bar{\gamma} \mathscr{A}_{I}^{2}$ [27]. Taking a fixed $R_{\mathrm{ddr}}=(10.5)^{1 / 2}$, the identical average energies of the in-phase and quadrature signals are obtained.

The impact of the parameter $R_{\mathrm{ddr}}$ is emerged to observe the system performance. When $R_{\mathrm{ddr}}=1$, the most favorable case occurs; this implies that the in-phase and quadrature distance are identical for both the Laplacian and Gaussian

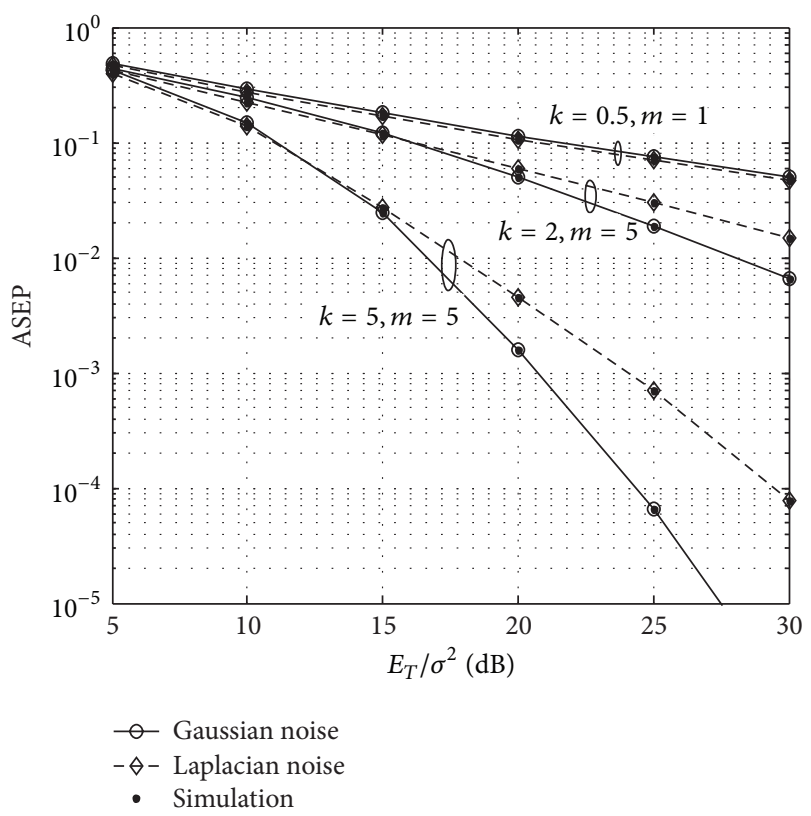

FIGURE 1: ASEP of $2 \times 2$ MIMO system using MMSE-OSIC over WG fading channel subject to Laplacian and Gaussian noise.

noise. For $R_{\mathrm{ddr}}=(10.5)^{1 / 2}$, same energy is obtained between the in-phase and quadrature signal; thus the system performance is reduced with a small amount, that is, approximately $1 \mathrm{~dB}$ SNR reduction for large SNRs. When the quadrature signal contains 10.5 times the average energy of the inphase signal, for this instant loss is more essential as it gets approximately $4 \mathrm{~dB}$ SNR loss for large SNRs, comparative to the aforementioned case, where $R_{\mathrm{ddr}}=1$.

Firstly, composite WG fading is considered in Laplacian noise environment. To obtain Weibull and Rayleigh fading, the parameters are settled to $m \rightarrow \infty$ and $m \rightarrow \infty$, $k=1$, respectively. Figure 1 depicts the ASEP as a function of average SNR per symbol $E_{T} / \sigma^{2}$ for both Gaussian and Laplacian cases of noise. In addition, distinct values of $m$ and $k$ are chosen to determine the severity of fading. Analytical results presented in this paper by (19) and (20) demonstrate the perfect match of the simulation results. The performance of the system is improved by increasing both the parameters $m$ and $k$. Results shown in Figure 1 illustrate that the ASEP performance in Laplacian noise is superior to that of Gaussian noise for lower SNR or less than $15 \mathrm{~dB}$ SNR. However, for high SNR, less fading $(k \geq 2)$, the situation is upturned and ASEP performance improves in the Gaussian noise compared with Laplacian noise. For severe fading $(m=0.5)$, Laplacian noise offers better results than Gaussian noise.

Afterward, Weibull and Rayleigh fading which are the special cases of WG fading are considered. In Figure 2, Rayleigh fading case is taken into account. In this case, system gives superior performance by diminishing $\eta$, which validates the previous result in which the Laplacian noise gives better performance than the Gaussian noise in severe fading. It is previously mentioned that large fading parameter refers to less fading. In Figure 3, the ASEP is demonstrated as a function of the SNR per QAM symbol in Weibull 


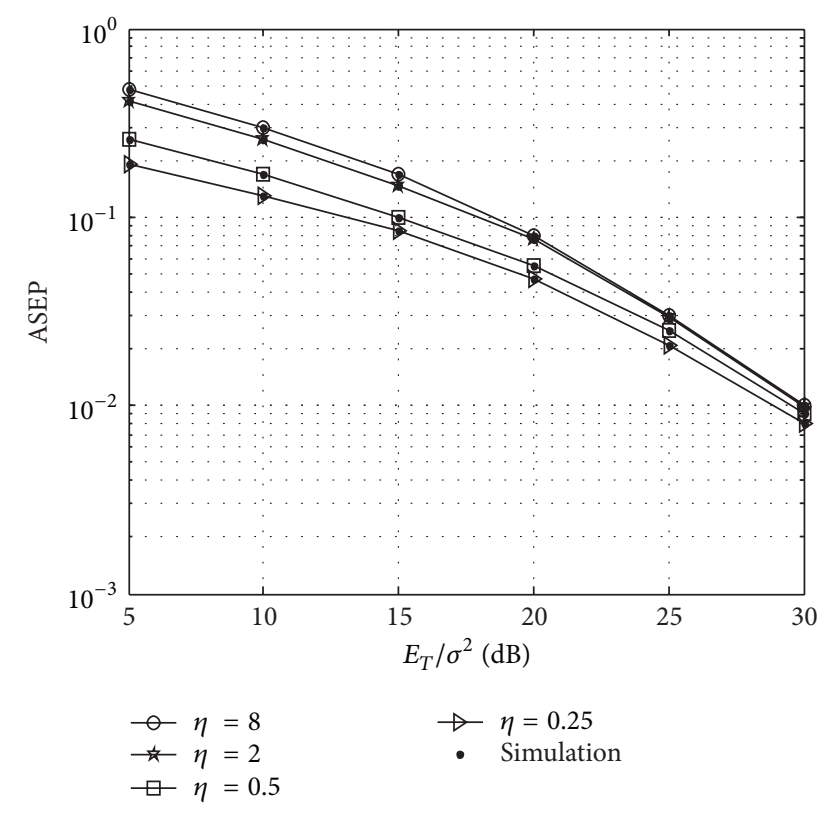

FIgURE 2: ASEP of $2 \times 2$ MIMO system for 16-QAM using MMSEOSIC over Rayleigh fading channel with arbitrary values of $\eta$.

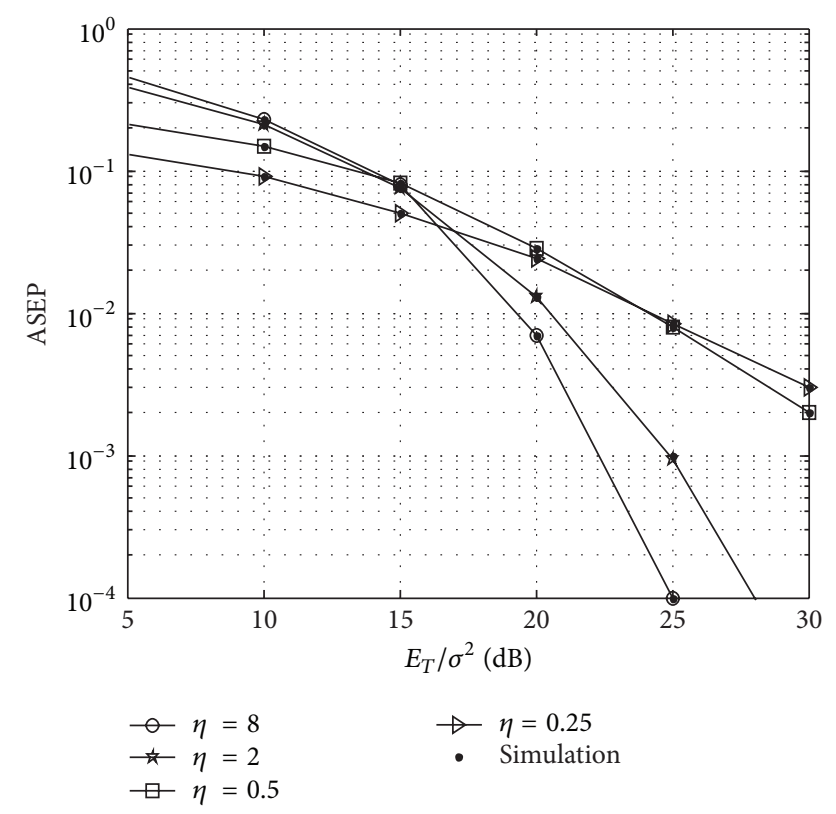

FIgURE 3: ASEP of $2 \times 2$ MIMO system for 16-QAM using MMSEOSIC detection over Weibull fading channel $(k=5)$ with arbitrary values of $\eta$.

fading environment $(k=5)$ with AWGGN for which $\eta=$ $8,2,0.5,0.25$. In this case, when the less fading condition occurs, the different two regions are investigated. At low SNR, the ASEP decreases with increasing $\eta$ and at high SNR it improves by increasing $\eta$.

\section{Conclusion}

This paper evaluates the ASEP performance of MIMO system in composite WG fading environment subject to AWGGN.
Analytical expressions for ASEP are derived using 16-QAM consisting of two independent in-phase and quadrature signals of PAM. The MMSE-OSIC detection is used to improve the error rate performance of MIMO system. It is concluded from the results that the ASEP performance in Laplacian noise is better than that of Gaussian noise for low SNR. However, in less fading, performance is degraded for high SNR and improved error performance is obtained in Gaussian noise compared with Laplacian noise. In severe fading, improved error rate performance can be achieved in the presence Laplacian noise compared with Gaussian noise. In Rayleigh fading case, the system gives superior performance for low noise shaping parameter $\eta$. This result again proves that the Laplacian noise gives better performance than the Gaussian noise in severe fading. In Weibull fading, the different two regions are inspected for lower amount of fading or large fading parameter. Moreover, the ASEP reduces with $\eta$ at low SNR and it increases by $\eta$ at high SNR. Simulation results validate the analytical results.

\section{Competing Interests}

The authors declare that there are no competing interests regarding the publication of this paper.

\section{References}

[1] E. Biglieri, R. Calderbank, A. Constantinides, A. Goldsmith, A. Paulraj, and H. V. Poor, MIMO Wireless Communications, Cambridge University Press, Cambridge, UK, 2007.

[2] Y. Lee, H.-C. Shih, C.-S. Huang, and J.-Y. Li, "Low-complexity MIMO detection: a mixture of ZF, ML and SIC," in Proceedings of the 19th International Conference on Digital Signal Processing (DSP '14), pp. 263-268, Hong Kong, August 2014.

[3] C. Siriteanu, S. D. Blostein, A. Takemura, H. Shin, S. Yousefi, and S. Kuriki, "Exact MIMO zero-forcing detection analysis for transmit-correlated rician fading," IEEE Transactions on Wireless Communications, vol. 13, no. 3, pp. 1514-1527, 2014.

[4] Y. Jiang, M. K. Varanasi, and J. Li, "Performance analysis of ZF and MMSE equalizers for MIMO systems: an in-depth study of the high SNR regime," IEEE Transactions on Information Theory, vol. 57, no. 4, pp. 2008-2026, 2011.

[5] H. Zhang, H. Dai, and B. L. Hughes, "Analysis on the diversitymultiplexing tradeoff for ordered MIMO SIC receivers," IEEE Transactions on Communications, vol. 57, no. 1, pp. 125-133, 2009.

[6] T.-H. Liu, "Some results for the fast MMSE-SIC detection in spatially multiplexed MIMO systems," IEEE Transactions on Wireless Communications, vol. 8, no. 11, pp. 5443-5448, 2009.

[7] Y. Song, C. Liu, and F. Lu, "Lattice reduction-ordered successive interference cancellation detection algorithm for multipleinput-multiple-output system," IET Signal Processing, vol. 9, no. 7, pp. 553-561, 2015.

[8] Y. Song, C. Liu, and F. Lu, "The optimal MMSE-based OSIC detector for MIMO system," IEICE Transactions on Communications, vol. E99-B, no. 1, pp. 232-239, 2016.

[9] Y. Bae and J. Lee, "Low complexity antenna selection for VBLAST systems with OSIC detection," EURASIP Journal on Wireless Communications and Networking, vol. 2011, article 6, 7 pages, 2011. 
[10] Y. S. Cho, J. Kim, W. Y. Yang, and C. G. Kang, MIMO-OFDM Wireless Communications with MATLAB, John Wiley \& Sons, 2010.

[11] P. M. Shankar, Fading and Shadowing in Wireless Systems, Springer, New York, NY, USA, 2012.

[12] T. Hashem and M. I. Islam, "Performance analysis of MIMO link under fading channels," in Proceedings of the 17th International Conference on Computer and Information Technology (ICCIT '14), pp. 498-503, Dhaka, Bangladesh, December 2014.

[13] A. Abdi and M. Kaveh, "K distribution: an appropriate substitute for Rayleigh-lognormal distribution in fading-shadowing wireless channels," Electronics Letters, vol. 34, no. 9, pp. 851-852, 1998.

[14] M. Matthaiou, N. D. Chatzidiamantis, G. K. Karagiannidis, and J. A. Nossek, "On the capacity of generalized- $K$ fading MIMO channels," IEEE Transactions on Signal Processing, vol. 58, no. 11, pp. 5939-5944, 2010.

[15] M. R. Bhatnagar and M. K. Arti, "On the closed-form performance analysis of maximal ratio combining in shadowed-rician fading LMS channels," IEEE Communications Letters, vol. 18, no. 1, pp. 54-57, 2014.

[16] M. R. Bhatnagar, "On the sum of correlated squared $\kappa-\mu$ shadowed random variables and its application to performance analysis of MRC," IEEE Transactions on Vehicular Technology, vol. 64 , no. 6, pp. 2678-2684, 2015.

[17] M. R. Bhatnagar and M. K. Arti, "Performance analysis of AF based hybrid satellite-terrestrial cooperative network over generalized fading channels," IEEE Communications Letters, vol. 17, no. 10, pp. 1912-1915, 2013.

[18] P. S. Bithas, "Weibull-gamma composite distribution: alternative multipath/shadowing fading model," Electronics Letters, vol. 45, no. 14, pp. 749-751, 2009.

[19] K. Tiwari and D. S. Saini, "SER improvisation of MIMO-MRC system over Weibull-Gamma fading channel," in Proceedings of the International Conference on Signal Processing and Communication (ICSC '15), pp. 70-73, IEEE, Noida, India, March 2015.

[20] N. C. Beaulieu, "A useful integral for wireless communication theory and its application to rectangular signaling constellation error rates," IEEE Transactions on Communications, vol. 54, no. 5, pp. 802-805, 2006.

[21] G. K. Karagiannidis, "On the symbol error probability of general order rectangular QAM in nakagami-m Fading," IEEE Communications Letters, vol. 10, no. 11, pp. 745-747, 2006.

[22] H. A. Suraweera and J. Armstrong, "A simple and accurate approximation to the SEP of rectangular QAM in arbitrary Nakagami-m fading channels," IEEE Communications Letters, vol. 11, no. 5, pp. 426-428, 2007.

[23] A. Mathur and M. R. Bhatnagar, "PLC performance analysis assuming bpsk modulation over nakagami- $m$ additive noise," IEEE Communications Letters, vol. 18, no. 6, pp. 909-912, 2014.

[24] A. Mathur, M. R. Bhatnagar, and B. K. Panigrahi, "PLC Performance analysis over Rayleigh fading channel under Nakagami$\mathrm{m}$ additive noise," IEEE Communications Letters, vol. 18, no. 12, pp. 2101-2104, 2014.

[25] A. Mathur, M. R. Bhatnagar, and B. K. Panigrahi, "Performance evaluation of PLC under the combined effect of background and impulsive noises," IEEE Communications Letters, vol. 19, no. 7, pp. 1117-1120, 2015.

[26] R. Viswanathan and A. Ansari, "Distributed detection of a signal in generalized Gaussian noise," IEEE Transactions on Acoustics, Speech, and Signal Processing, vol. 37, no. 5, pp. 775$778,1989$.
[27] H. Soury, F. Yilmaz, and M.-S. Alouini, "Error rates of M-PAM and M-QAM in generalized fading and generalized gaussian noise environments," IEEE Communications Letters, vol. 17, no. 10, pp. 1932-1935, 2013.

[28] M. A. Chaudhry and S. M. Zubair, On a Class of Incomplete Gamma Functions with Applications, Chapman \& Hall/CRC, Boca Raton, Fla, USA, 1st edition, 2002.

[29] M. Nardon and P. Pianca, "Simulation techniques for generalized Gaussian densities," Working Papers 145, Departement of Applied Mathematics, Università Ca' Foscari Venezia, 2006.

[30] F. Yilmaz and M.-S. Alouini, "A new simple model for composite fading channels: second order statistics and channel capacity," in Proceedings of the 7th International Symposium on Wireless Communication Systems (ISWCS '10), pp. 676-680, IEEE, York, UK, September 2010.

[31] A. A. Kilbas and M. Saigo, H-Transforms: Theory and Applications (Analytical Methods and Special Functions), CRC Press, New York, NY, USA, 1st edition, 2004.

[32] H. Soury, F. Yilmaz, and M.-S. Alouini, "Average bit error probability of binary coherent signaling over generalized fading channels subject to additive generalized Gaussian noise," IEEE Communications Letters, vol. 16, no. 6, pp. 785-788, 2012.

[33] A. P. Prudnikov, Y. A. Brychkov, and O. I. Marichev, Integrals and Series: More Special Functions, vol. 3, Gordon and Breach Science Publishers, New York, NY, USA, 1990.

[34] G. D. Golden, C. J. Foschini, R. A. Valenzuela, and P. W. Wolniansky, "Detection algorithm and initial laboratory results using V-BLAST space-time communication architecture," Electronics Letters, vol. 35, no. 1, pp. 14-15, 1999.

[35] N. C. Beaulieu, "A useful integral for wireless communication theory and its application to rectangular signaling constellation error rates," IEEE Transactions on Communications, vol. 54, no. 5, pp. 802-805, 2006.

[36] P. K. Mittal and K. C. Gupta, "An integral involving generalized function of two variables," Indian Academic Science, vol. 75, pp. 117-123, 1972. 


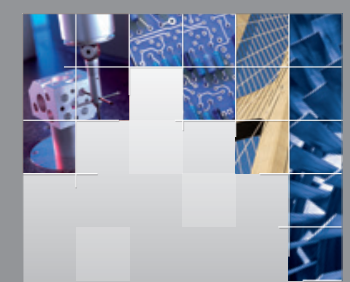

\section{Enfincering}
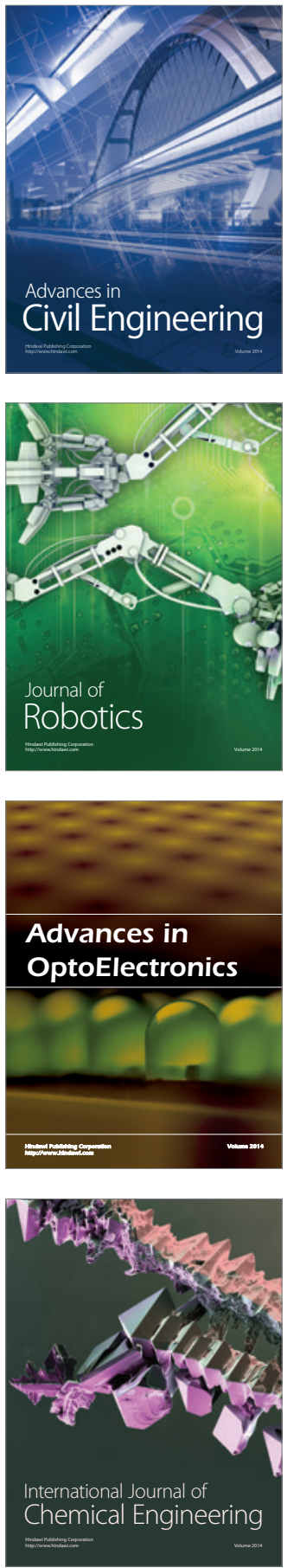

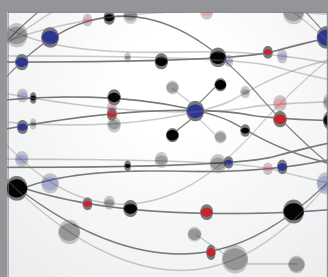

The Scientific World Journal

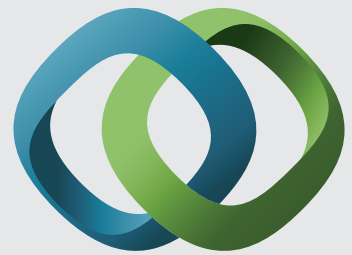

\section{Hindawi}

Submit your manuscripts at

http://www.hindawi.com
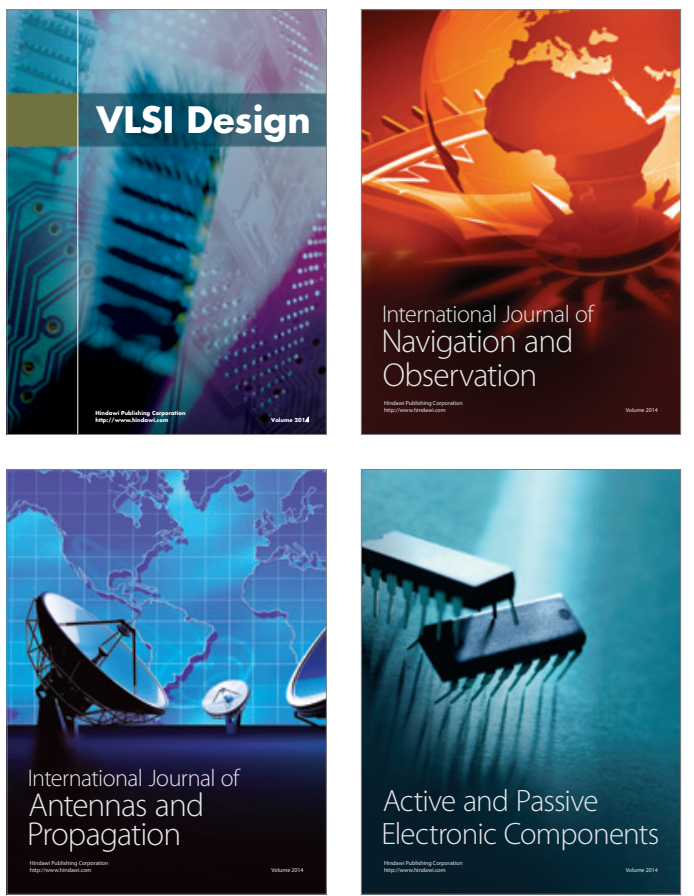
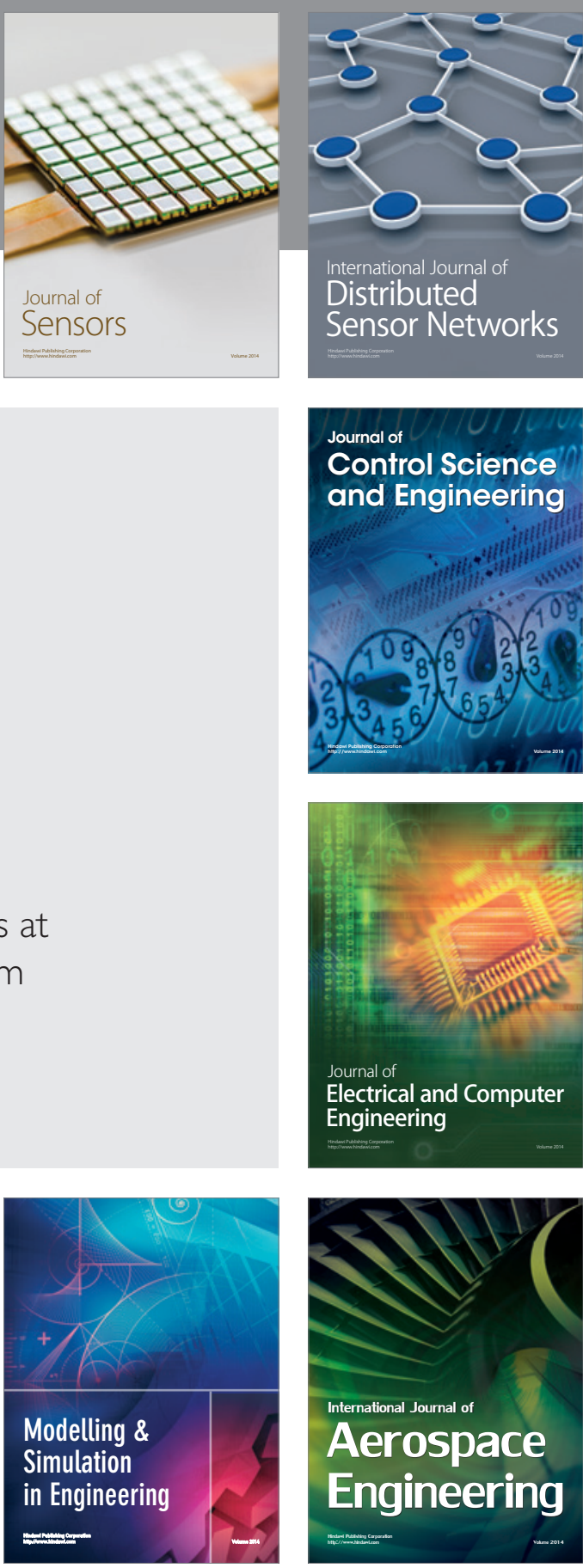

International Journal of

Distributed

Sensor Networks

Journal of

Control Science

and Engineering
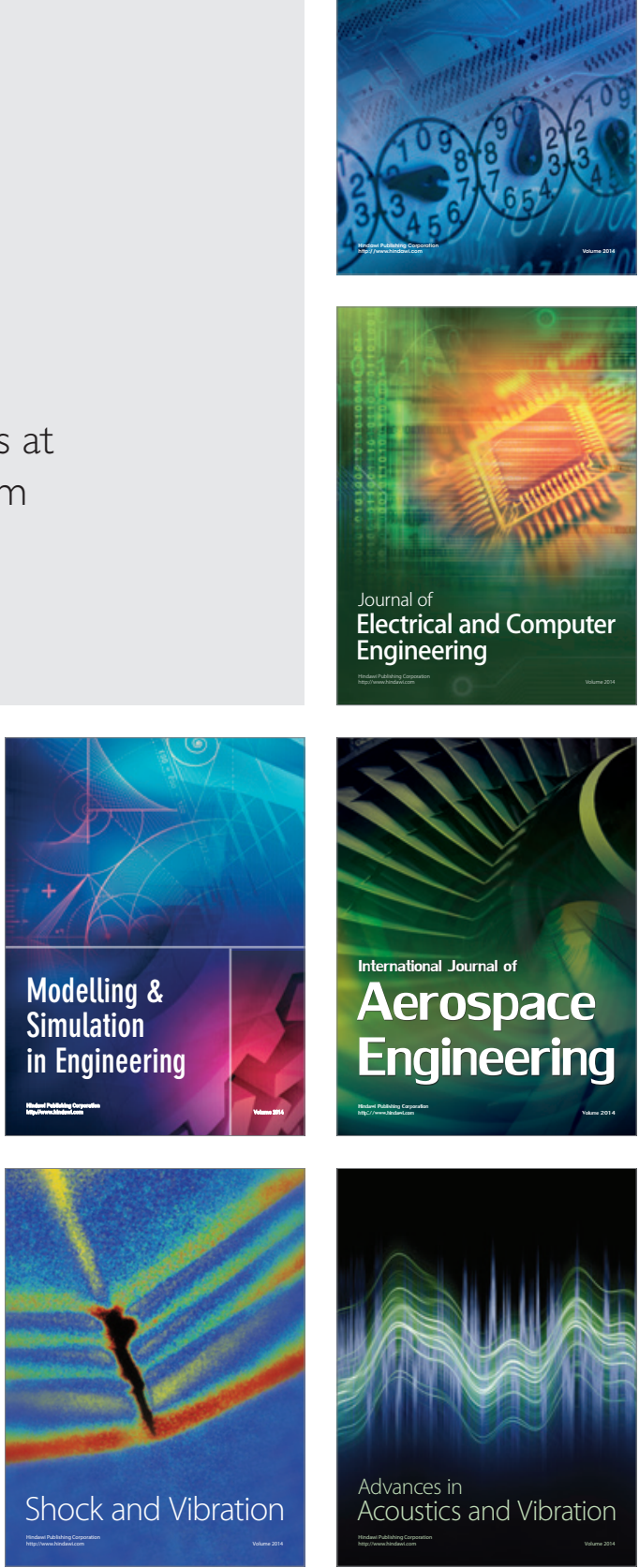